\title{
Thermally induced self-healing epoxy/glass laminates with porous layers containing crystallized healing agent
}

\author{
T. Szmechtyk ${ }^{*}$, N. Sienkiewicz, K. Strzelec \\ Institute of Polymer and Dye Technology, Faculty of Chemistry, Lodz University of Technology, Stefanowskiego 12/16, \\ 90-924 Łodz, Poland
}

Received 20 December 2017; accepted in revised form 19 February 2018

\begin{abstract}
Porous glass fiber and paper layers were tested for application in thermally induced self healing epoxy laminates as healing porous layers. Both types of layers were impregnated using high purity bisphenol A diglycidyl ether (BADGE) epoxy with ability to crystallize during storage under $25^{\circ} \mathrm{C}$. Absorption capacity of porous layers was evaluated. Differential scanning calorimetry was used to investigate BADGE healing agent recrystallization process. Healing porous glass layers (HPGL) were selected for further tests. Liquid chromatography and Fourier transform infrared (FT IR) spectroscopy provided information about average molecular mass of embedded healing agent and functional groups in HPGL layers. Self-healing efficiency of three different laminates with HPGL layers was calculated based on the results of three-point bending test and Charpy impact test. Also, flexural properties and impact strength of laminates were evaluated. The obtained results confirm competitive self healing ability of composites with HPGL.
\end{abstract}

Keywords: thermosetting resins, epoxy/glass composites, self-healing, porous laminate layers

\section{Introduction}

Self-healing of polymer materials is an approach, which focuses on counteracting the effects of mechanical fracture during its use. Extrinsic self-healing can be provided by three types of systems: microcapsules [1], hollow fibers [2] and microvascular networks [3]. Microcapsules are a simple, mostly one time use solution which consist in releasing a healing agent as a result of microcrack propagation. The main drawback of this system is lack of a healing agent in the repaired area. Subsequent stress may cause secondary microcrack propagation in the area and consequently critical fracture, especially since the healed area is usually more susceptible to secondary stress than a virgin matrix. Hollow fibers and microvascular networks partially solve the one-time use problem with elongation of the container. This increases the probability of unreacted healing agent presence in cracked containers and provides secondary self-healing. Microvascular networks can also be refilled with a reactive agent. On the other hand, selfhealing from hollow fibers and microvascular networks is still limited by the fiber or channel diameter. Each healing process increases the risk of tube sealing while stopping the healing agent flow. Consequently, secondary or tertiary healing can still fail in the same area. Mixed systems, such as the glass fiber reinforced epoxy composite with microcapsules filled with dicyclopentadiene (DCPD), have their own limitations: cracks inside fiber have no access to DCPD and cannot be healed [4]. Other systems implemented in epoxy/glass composites (EGC) are also imperfect [5]. Zako and Takano [6] impregnated microparticles of thermoplastic adhesive in EGC and obtained a system which was able to heal at $120^{\circ} \mathrm{C}$; however, embedded particles left empty spaces, 
which lowered composite integrity. A similar solution has been developed for shape memory polymers, which are gaining in popularity. Yao et al. [7] and Karger-Kocsis [8] obtained epoxy/poly( $\varepsilon$-caprolactone) (PCL) blends (Yao, $14 \mathrm{wt} \%$ of PCL electrospun web, partly broken specimens; Karger-Kocsis, various wt $\%$ of PCL with different $T_{\mathrm{g}}$ epoxies, fully broken specimens) with self-healing ability. PCL as the thermoplastic healing agent provides effective selfhealing under $80^{\circ} \mathrm{C}$, but its use in EGC is hampered by reduced ductility and deformability of the reinforced composite, which limits the design flexibility for temporary shaping [9]. Trask and Bond obtained EGC with lattice of hollow fibers with optimized degrees of crossing and healing agent separated from hardener in different fibers [10]. Despite the very good results $(87 \%$ residual strength of reference laminate and self-healing efficiency slightly above $100 \%$ ), there is no data concerning secondary self-healing. Contact points between epoxy healing agent and hardener are limited as well, which may hinder secondary healing. The latest systems attempt to eliminate these drawbacks. Zhang group developed polypropylene tubes with pressurized mercaptan and epoxy healing agents [11]. Higher pressure was obtained with a foaming agent and improved self-healing efficiency with increased flowability of the healing agent. However, longer foaming time and gas leakage from the tubes after storage caused a decrease in composite durability. Yildiz and co-workers applied a self healing system with fibers made of poly(methyl methacrylate) and polyacrylamide as an outer and inner layer, respectively [12]. These fibers containing the healing agent were produced using tri-axial electrospinning and used to obtain EGC with the selfhealing system. This solution is an alternative for glass fiber based systems, but does not attempt to solve the multiple healing issue. Bekas et al. [13] focused on modifying a healing agent: epoxy doped with Multi-Wall Carbon Nanotubes (MWCNTs) was applied in a microvascular system. A nanoreinforcement effect was observed after self-healing and efficiency of first healing clearly exceeded $100 \%$. Kostopoulos et al. [14] applied interleaves of supramolecular polymer for epoxy/carbon fiber laminate and reported multiple recovery, even after the seventh cycle. The remaining two of the above-mentioned extrinsic systems seem to be the most advanced and effective of the recent solutions. Although the intrinsic self-healing systems in epoxy composites are not quite popular, there are also several recent systems worth mentioning. Lu et al. [15] proposed an epoxy system based on transesterification reaction in the presence of zinc acetylacetonate hydrate catalyst, but its main limitations are the high temperature $\left(150^{\circ} \mathrm{C}\right)$ and pressure $(6 \mathrm{MPa})$ requirements for self-healing. Less demanding conditions $\left(70-85^{\circ} \mathrm{C}\right)$ were needed for the self-healing system based on disulphide by Post et al. [16]. However, recent advances show that thermoset networks based on dynamic covalent bond chemistry have already achieved the mechanical properties of conventional thermosets.

In this study, we present a novel type of thermally induced self-healing system for EGC, which contains solid healing agent in porous layers. High purity bisphenol A diglycidyl ether (BADGE) epoxy was selected as the healing agent because of its ability to crystallize in temperature under $25^{\circ} \mathrm{C}$. BADGE was embedded in porous glass fiber layers and crystallized to obtain a solid healing agent. According to our knowledge, there is no system for EGC which uses pores as containers for a crystallized healing agent.

\section{Experimental}

\subsection{Materials}

D.E.R. 332 epoxy resin (high purity bisphenol A diglycidyl ether with epoxy equivalent weight $171-$ $175 \mathrm{~g} / \mathrm{eq}$ and viscosity $4000-6000 \mathrm{mPa} \cdot \mathrm{s}$ at $25^{\circ} \mathrm{C}$ ) was purchased from Sigma-Aldrich. Triethylenetetramine (TETA) was obtained from Organika-Sarzyna Inc. (Poland). Whatman glass microfiber filters, grade GF/A were purchased from Labo Plus (Poland). Filtrak 390 paper filters were acquired from Avantor Performance Materials (Poland). The woven glass fiber powder mats (density of $300 \mathrm{~g} / \mathrm{m}^{2}$ ) were supplied by Krosglass S.A. (Poland).

\subsection{Preparation of porous layers with healing agent}

GF/A and Filtrak 390 filters (filters parameters are presented in Table 1) were immersed in liquid D.E.R. 332 epoxy and impregnated using wet vacuum impregnation process. The obtained vacuum was $12 \mathrm{kPa}$ and filters were soaked in resin bath for 10 minutes to remove air from pores. Then, the vacuum was removed and filters were left for another 10 minutes to absorb epoxy into pores. Excess of unabsorbed epoxy was removed and filters were left on tefloned fabric, at a temperature of $20^{\circ} \mathrm{C}$ to crystallize. After 3 days, filters with crystallized epoxy - healing 
Table 1. Filters parameters.

\begin{tabular}{|l|c|c|c|}
\hline \multicolumn{1}{|c|}{ Filter type } & $\begin{array}{c}\text { Particle } \\
\text { retention } \\
{[\boldsymbol{\mu \mathbf { m } ]}}\end{array}$ & $\begin{array}{c}\text { Thickness } \\
{[\boldsymbol{\mu \mathbf { m } ]}}\end{array}$ & $\begin{array}{c}\text { Weight } \\
{\left[\mathbf{g} / \mathbf{m}^{2}\right]}\end{array}$ \\
\hline Filtrak 390 paper filter & $3-5$ & 160 & 84 \\
\hline Whatman GF/A glass filter & 1.6 & 260 & 53 \\
\hline
\end{tabular}

porous layers were carefully detached from the tefloned surface.

\subsection{Gravimetric analysis of porous layer absorption capacity}

GF/A and Filtrak 390 filters were weighed before impregnation. Healing porous glass layers (HPGL) obtained from GF/A filters and healing porous paper layers (HPPL) obtained from Filtrak 390 filters were weighed after epoxy resin crystallization. Ten samples of each filter type were tested and averaged. The samples were weighed using laboratory scales with an accuracy of $0.1 \mathrm{mg}$. Porous layer absorption capacity $(P L A C)$ was calculated according to Equation (1):

$$
P L A C=\frac{m_{\mathrm{I}}-m_{\mathrm{N}}}{m_{\mathrm{N}}} \cdot 100[\%]
$$

where $P L A C$ - porous layer absorption capacity, $m_{\mathrm{N}}$ - mass of neat filter, $m_{\mathrm{I}}-$ mass of impregnated filter.

\subsection{Preparation of epoxy composites with single healing porous layers for preliminary tests}

Three types of healing porous layers were prepared: HPGL layers, HPPL layers, and HPPL layers covered with acrylic matt varnish (ACHPPL) for improved healing agent protection All healing porous layers were covered on both sides with thin layer of D.E.R. 332 epoxy mixed with excess of TETA (16 phr instead of 14 close to equimolar for self-healing reaction in mild conditions) and placed between two tefloned fabrics to obtain less than $1 \mathrm{~mm}$ thick layer of the composite. A similar layer of cured epoxy (with $14 \mathrm{phr}$ of TETA, for amine/epoxy equimolar ratio) without healing porous layers was prepared as reference (REF).

\subsection{Differential scanning calorimetry (DSC)}

Self-healing process of the composites with single healing porous layers was studied using a TGA/DSC1 (Mettler Toledo) analyzer. Samples were cooled to $-50{ }^{\circ} \mathrm{C}$ and then heated to $200^{\circ} \mathrm{C}$ (both in an argon atmosphere) at a heating rate of $10^{\circ} \mathrm{C} / \mathrm{min}$. DSC curves of composites and reference were compared (from 0 to $190^{\circ} \mathrm{C}$ ). Two cycles were conducted for each sample to evaluate DSC curve after post-curing.

\subsection{Preparation of epoxy composite with HPGL layers stained with carbon black}

HPGL layers were selected as most promising porous layers for self-healing composites. Single rectangular specimen with one HPGL layer (stained with carbon black) was prepared for observation of healing agent release at elevated temperature. Photograph of healing agent release was taken.

\subsection{Liquid chromatography (LC)}

Retention time $\left(r_{\mathrm{T}}\right)$ of the healing agent from HPGL layer was compared with $r_{\mathrm{T}}$ of D.E.R. 332 reference and were measured using an HP 1100 series liquid chromatograph, equipped with PLgel 5 m Mixed$\mathrm{C}$ column. Tetrahydrofuran (THF) was used as an eluent at flow rate $0.5 \mathrm{~mL} / \mathrm{min}$. A single HPGL layer sample was immersed in THF for 48 hours at room temperature to rinse D.E.R. 332 healing agent to THF.

\subsection{Fourier transform infrared (FT-IR) spectroscopy}

The FT-IR spectra of the epoxy composite with single HPGL layer and reference were collected in an air atmosphere using Nicolet 6700 FT-IR spectrophotometer (Thermo Scientific, USA) equipped with a diamond crystal. Spectra were taken three times, at room temperature in a range of $400-4000 \mathrm{~cm}^{-1}$, each from a different region of the sample. OMNIC Spectra tool was used for ATR correction and bands scales normalization.

\subsection{Preparation of epoxy/glass composites with HPGL layers for mechanical tests}

Appropriate pieces of HPGL layers have been placed alternately in a silicone mold with similar pieces of woven glass fiber mats reinforcement in a sandwich structure to obtain unnotched, rectangular specimens $(80 \times 10 \times 4 \mathrm{~mm})$. All layers were separated from each other using a mixture of D.E.R. 332 epoxy resin and TETA hardener (16 and $20 \mathrm{phr}$ ). Reference samples ( 1 - without glass layers; 2 - only with HPGL layers) were also prepared. Scheme of the composites structures is presented in Figure 1. Composition of all prepared samples is listed in Table 2. All composites were cured in $50^{\circ} \mathrm{C}$ for 72 hours. 
Table 2. Composition of laminates.

\begin{tabular}{|c|l|l|c|c|c|c|}
\hline No. & \multicolumn{1}{|c|}{ Composite } & Short name & $\begin{array}{c}\text { D.E.R. 332 } \\
\text { [phr] }\end{array}$ & $\begin{array}{c}\text { TETA } \\
\text { [phr] }\end{array}$ & $\begin{array}{c}\text { Woven glass } \\
\text { fiber mats }\end{array}$ & $\begin{array}{c}\text { Healing porous } \\
\text { glass layers }\end{array}$ \\
\hline 0 & reference sample & REF 14 & 100 & 14 & - & - \\
\hline 1 & laminate with HPG layers without reinforcement & HPGL 16 & 100 & 16 & - & + \\
\hline 2 & laminate 1 with HPG layers and reinforcement & HPGL RF 16 & 100 & 16 & + & + \\
\hline 3 & laminate 2 with HPG layers and reinforcement & HPGL RF 20 & 100 & 20 & + & + \\
\hline
\end{tabular}

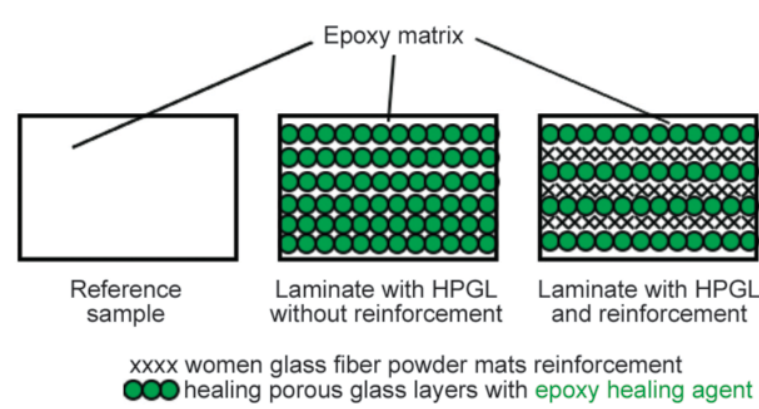

Figure 1. Scheme of composites structures.

\subsection{Three-point bending test}

A three-point bending test of self-healing efficiency was conducted on Zwick/Roell 1435 universal testing machine (Germany), according to ISO 178. Rectangular specimens $(80 \times 10 \times 4 \mathrm{~mm})$ without a notch were bent at room temperature, at the testing speed of $2 \mathrm{~mm} / \mathrm{min}$. All composites and reference sample were tested sixfold. Protective splints were used to cover both ends of specimen and left small, central part with crack tip exposed. It allowed subsequent failures to follow the initial crack path [17]. After failure, all test bars (except reference) were restored carefully to their original shape with pushing together the splints and placed at $50^{\circ} \mathrm{C}$ for 48 hours for thermally induced self-healing. Healed specimens were bent until failure and restored again. Healing at $50{ }^{\circ} \mathrm{C}$ was also repeated. The obtained results of maximum flexural stress $\left(\sigma_{\mathrm{fM}}\right)$ and flexural modulus $\left(E_{\mathrm{f}}\right)$ for virgin and healed composites after each of the three healing cycles were expressed as mean values and compared to quantify self-healing efficiency ( $S H E$ ), which was calculated according to Wool and O'Connor equation (1) [18] (Equation (2)):

$S H E=\frac{P_{\mathrm{H}}}{P_{\mathrm{V}}} \cdot 100[\%]$ where $S H E$ - self-healing efficiency, $P_{\mathrm{H}}$ - property of healed composite, $P_{\mathrm{V}}-$ property of virgin composite.

\subsection{Charpy impact test}

Charpy impact test was carried out on Cometech QC639P/Q universal impact tester (Taiwan), according to ISO 179. The identical rectangular specimens $(80 \times 10 \times 4 \mathrm{~mm})$, also without a notch, were broken with a $2 \mathrm{~J}$ pendulum hammer. Impact strength results of the two sets were compared. The first set was examined after three-point bending test (restored to original shape with the splints to follow the initial crack path) and 48 hours of $50^{\circ} \mathrm{C}$ self-healing. The second set was tested directly to obtain reference values of virgin material. Self-healing efficiency was calculated according to self healing efficiency equation as for three-point bending test.

\section{Results and discussion}

\subsection{Gravimetric analysis of porous layer absorption capacity}

Averaged masses of both neat and impregnated filters and calculated porous layer absorption capacities are presented in Table 3. Absorption capacity of Whatman GF/A filter exceeds three times absorption capacity of Filtrak 390 filter. Mass of epoxy absorbed by Whatman GF/A filter is over eight times greater than the mass of the filter, while the mass of epoxy absorbed by Filtrak 390 filter is only two and half times greater than the mass of the filter. It clearly shows greater self-healing potential of HPGL layers.

\subsection{Differential scanning calorimetry (DSC)}

Comparison of DSC curves shows how composites behave at elevated temperatures (Figure 2). For the more detailed scheme of layer structure see Figure 8. All samples in the first cycle have an endothermic

Table 3. Porous layer absorption capacity.

\begin{tabular}{|l|c|c|c|}
\hline \multicolumn{1}{|c|}{ Filter type } & $\begin{array}{c}\text { Mass of neat filter } \\
{[\mathbf{m g}]}\end{array}$ & $\begin{array}{c}\text { Mass of impregnated filter with D.E.R. 332 } \\
{[\mathbf{m g}]}\end{array}$ & $\begin{array}{c}\text { Porous layer absorption capacity } \\
{[\%]}\end{array}$ \\
\hline Filtrak 390 paper filter & 13.2 & 45.3 & 243.2 \\
\hline Whatman GF/A glass filter & 76.0 & 710.3 & 834.6 \\
\hline
\end{tabular}



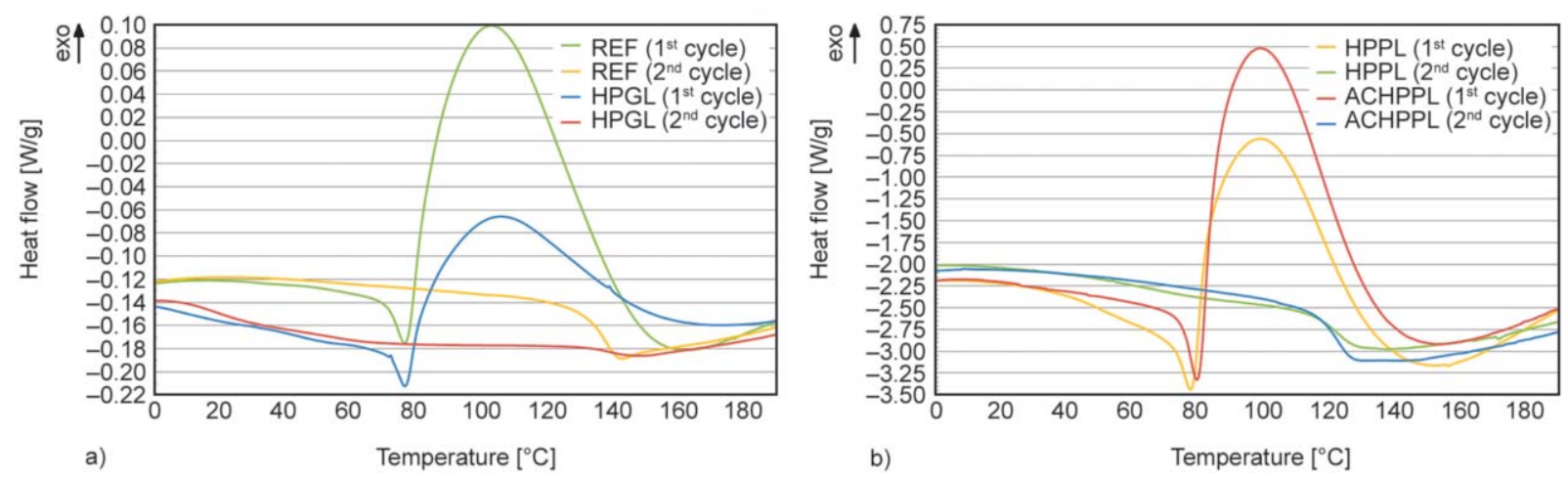

Figure 2. First and second cycle DSC curves: (a) reference and HPGL composite, (b) HPPL and ACHPPL composites.

peak around $75^{\circ} \mathrm{C}$, which indicates melting of the cured epoxy matrix from the external layer, and exothermic region of post-curing with a peak around $110^{\circ} \mathrm{C}$ (also external layer). A difference between reference and samples with healing porous layers (HPL) is observed in the range of $0-70{ }^{\circ} \mathrm{C}$; the presence of inflection points on baseline indicates glass transition temperatures of healing agent fractions from HPL, which also contain amorphous phase. The highest $T_{\mathrm{g}}$ inflexion point in this region is assigned to cured epoxy and is observed close to the melting peak. A lower $T_{\mathrm{g}}$ accompanies lower molecular mass fractions of D.E.R. 332 epoxy from HPL [19]. This correlation provides information, that HPGL single layer composite have the highest level of internal solid healing agent among all the tested composites [20]. The similar region in the second cycle is a proof of the healing agent stability in this system. It also shows that the healing agent in liquid phase does not react completely with TETA excess from external layers if no mechanical factor occurs. More differences of the $0-70^{\circ} \mathrm{C}$ range between both cycles are observed at the HPPL and ACHPPL curves (Figure 2b), meaning that these layers are less stable. In addition to this, both paper-based systems have less pronounced inflection points in comparison with HPGL. The great performance of HPGL makes it a viable option for further tests.

\subsection{Stained healing agent release photograph}

Specimen with single HPGL layer stained with carbon black was broken and heated to $50^{\circ} \mathrm{C}$. Appreciable amount of stained healing agent (D.E.R. 332 epoxy) leaked from the specimen with single HPGL (Figure 3). More layers of HPGL would provide enough healing agent for better sealing multiple times.

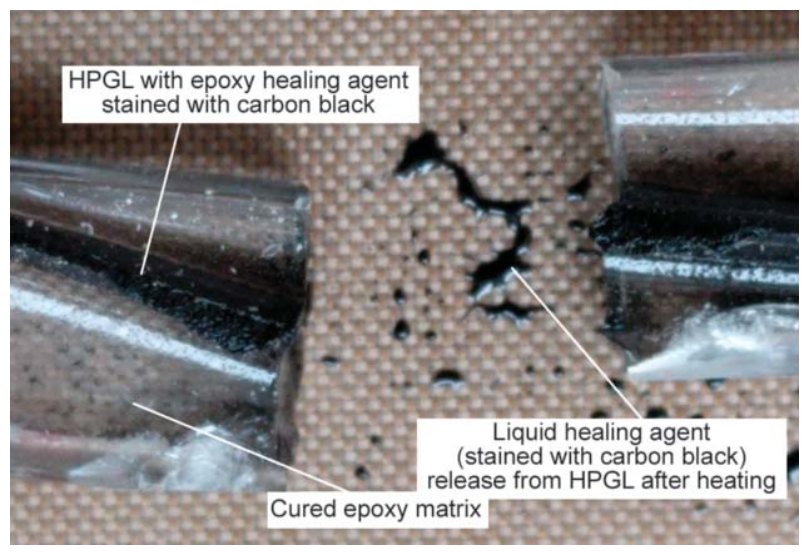

Figure 3. Photograph of composite with stained HPGL layers: elevated temperature causes healing agent release from broken parts of specimen.

\subsection{Liquid chromatography}

Liquid chromatography of neat BADGE and healing agent fractions rinsed out from HPGL were compared to investigate the molar mass of the healing agent. Both retention times of reference D.E.R. 332 and the healing agent from HPGL are similar (Figure 4). It confirms the presence of epoxy, which has a high content of BADGE with an initial molecular mass equal to D.E.R. 332. This indicates that the healing agent from glass porous layers is still serviceable

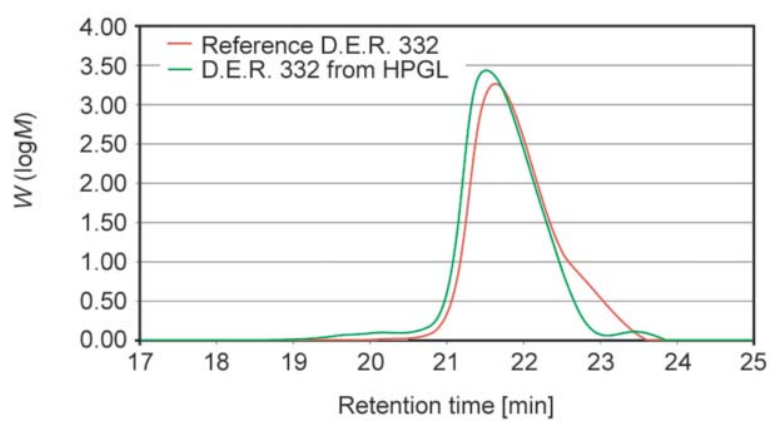

Figure 4. Liquid chromatogram of reference D.E.R. 332 and healing agent from HPGL. 


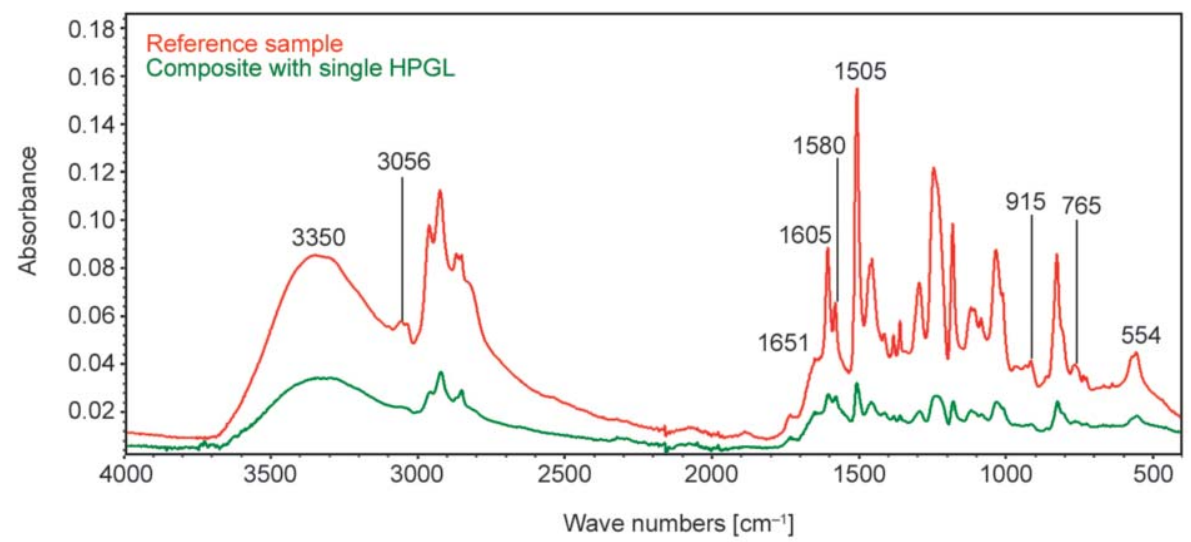

Figure 5. FT-IR spectra of reference sample and composite with single HPGL.

after composite cross-linking. Low molecular mass of the healing agent also will result in higher mobility of its molecules and, in consequence, better reactivity during a self healing process.

\subsection{Fourier transform infrared (FT-IR) spectroscopy}

Band intensities of the reference composite (REF) and single HPGL composite were compared (Figure 5). Band intensity ratios (HPGL/REF) of aromatic ring bands being resin's internal references vary from 0.205 for stretching of $\mathrm{C}=\mathrm{C}\left(1505 \mathrm{~cm}^{-1}\right)$ to 0.318 for in-plane deformation bending of $\mathrm{C}-\mathrm{H}$ $\left(1605 \mathrm{~cm}^{-1}\right)$ [21, 22]. HPGL/REF band intensity ratios of epoxy ring characteristic groups are 0.380 and 0.354 for 3056 and $915 \mathrm{~cm}^{-1}$, respectively. Also, HPGL/REF band intensity ratios from amine hardener $\left(3350,1651,1580,765\right.$ and $\left.554 \mathrm{~cm}^{-1}\right)$ are higher $(>0.400)$ than reference ratios from the aromatic ring. It is a result of the $16 \mathrm{phr}$ ratio of the TETA hardener, which remains unreacted despite the presence of the epoxy healing agent in HPGL. The slightly lower ratio of epoxy groups is the effect of low penetration depth of the IR beam in ATR mode.

\subsection{Three-point bending test}

Self-healing properties of three HPGL composites were examined. Also, flexural modulus $\left(E_{\mathrm{f}}\right)$ and maximum flexural stress $\left(\sigma_{\mathrm{fM}}\right)$ of virgin samples were compared for reference (Figure 6). All the composites with HPGL have lower flexural strength than reference, which might be caused by the defects of the laminate structure and the so-called eggshell effect. Despite these limitations, a virgin sample of the reinforced HPGL composite cured with $20 \mathrm{phr}$ of amine hardener is only slightly worse $(81.1 \%$ of sample REF $14 E_{\mathrm{f}}$ and $90.0 \%$ of sample REF $14 \sigma_{\mathrm{fM}}$ ). Flexural strength of composite HPGL RF 16 is also satisfactory (70.3 and $65.5 \%$ compared to the reference REF 14). Sample HPGL 16 without reinforcement, as expected, has the lowest values of flexural parameters (43.5 and $17.6 \%$ compared to sample REF 14).

On the other hand, sample HPGL 16 has over 100\% $E_{\mathrm{f}}$-based efficiency of the first self-healing, but only $52 \%$ recovery of $\sigma_{\mathrm{fM}}$. Next $S H E \mathrm{~s}$ of composite HPGL 16 fall sharply, which is caused by the absence of reinforcement. Composite HPGL RF 20 does not retain flexural properties after subsequent self-healing either, probably suffering from too large excess of amine hardener and brittleness, consequently. The best performance is exhibited by the HPGL RF 16 composite with good flexural properties and satisfactory subsequent self-healing (SHE $E_{\mathrm{f}}: 1^{\text {st }}-80.5 \%$; $2^{\text {nd }}-53.0 \%, 3^{\text {rd }}-40.5 \% ;$ SHE $\sigma_{\mathrm{fM}}: 1^{\text {st }}-63.9 \% ; 2^{\text {nd }}$ $\left.-36.3 \%, 3^{\text {rd }}-20.1 \%\right)$. According to Barbero et al. [23] and Privman et al. [24] continuum definition of healing efficiency is appropriate.

\subsection{Charpy impact test}

Charpy impact test results clearly show the influence of reinforcement on impact strength $(U)$ (Figure 7). The impact strength of both reinforced composites exceeds twice reference value, while $U$ of sample HPGL 16 is ten times smaller. Laminates HPGL RF 16 and HPGL RF 20 have similar performance to flexural - virgin composite with a higher excess of amine hardener is slightly better, but specimens with $16 \mathrm{phr}$ of TETA have higher self healing efficiency (63.7 to 53.7\%). Similar SHE $U$ of sample HPGL 16 $(60.4 \%)$ is meaningless at its low values of impact strength. 

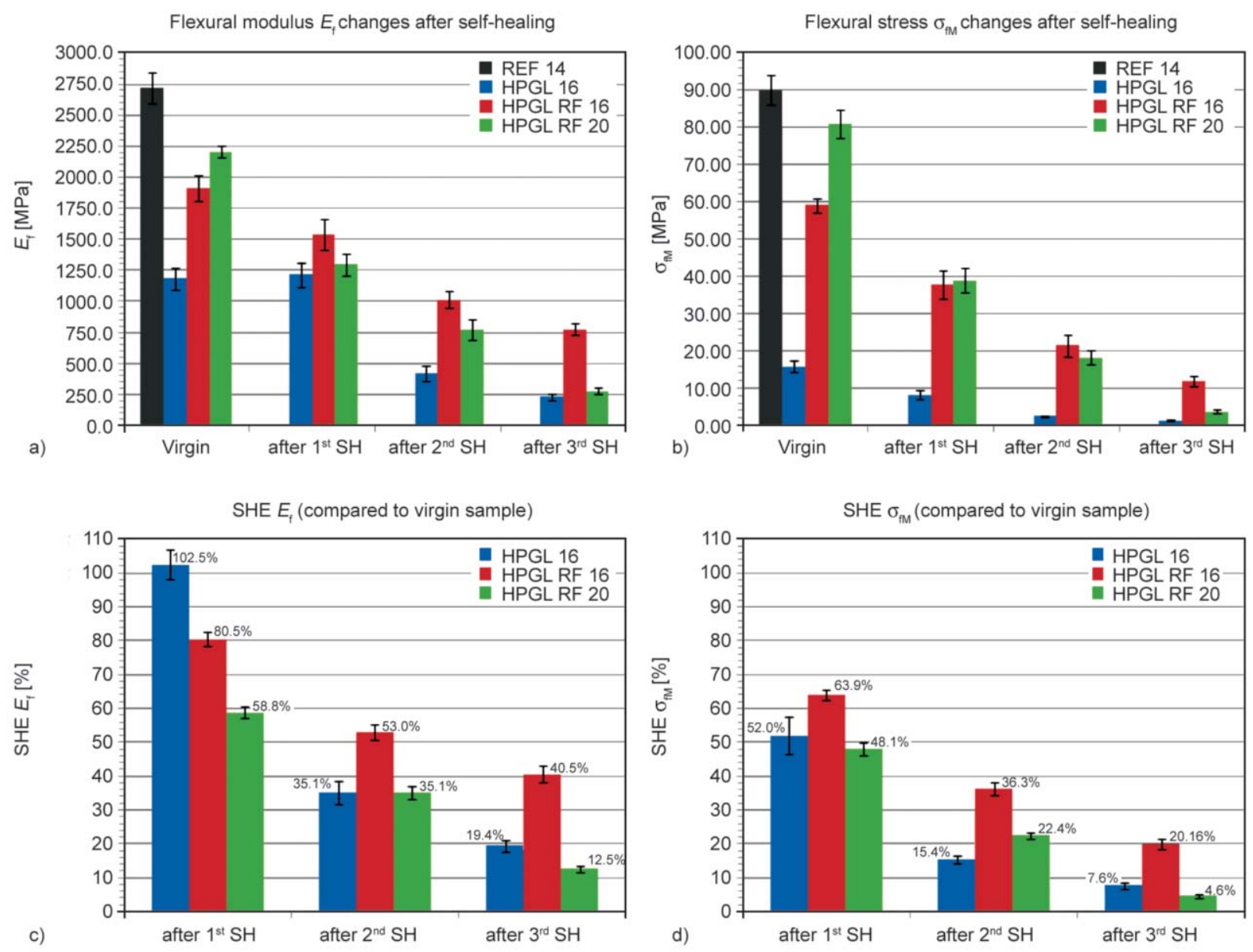

Figure 6. Comparison of three-point bending test parameters: flexural modulus $E_{\mathrm{f}}$ changes after self healing (a) and selfhealing efficiency based on $E_{\mathrm{f}}(\mathrm{c})$; flexural stress $\sigma_{\mathrm{fM}}$ changes after self-healing (b) and self-healing efficiency based on $\sigma_{\mathrm{fM}}(\mathrm{d})$.

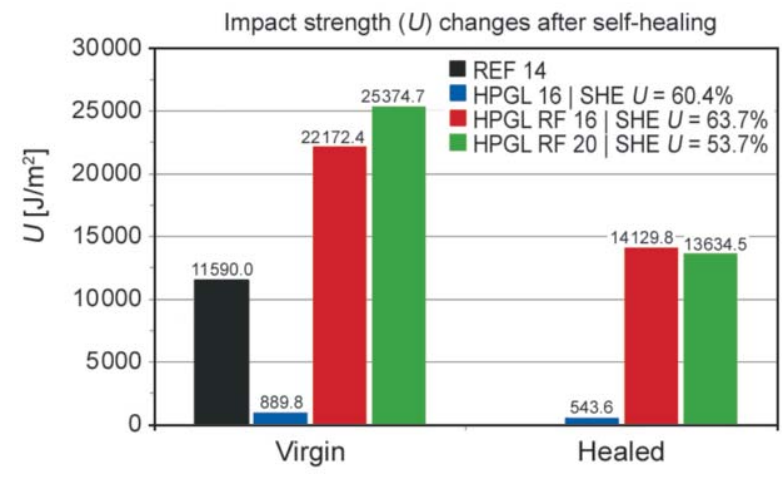

Figure 7. Comparison of Charpy impact test results.

\subsection{Discussion}

Both mechanical tests were conducted to reach the maximum potential of the composites. The self-healing system had to recover critical crack instead of few microcracks. The results obtained show, that it will be possible to repair small microcracks quite easily and without impacting the original shape. In consequence, in a normal situation (microcrack propagation during a standard use), there will be no need to manually

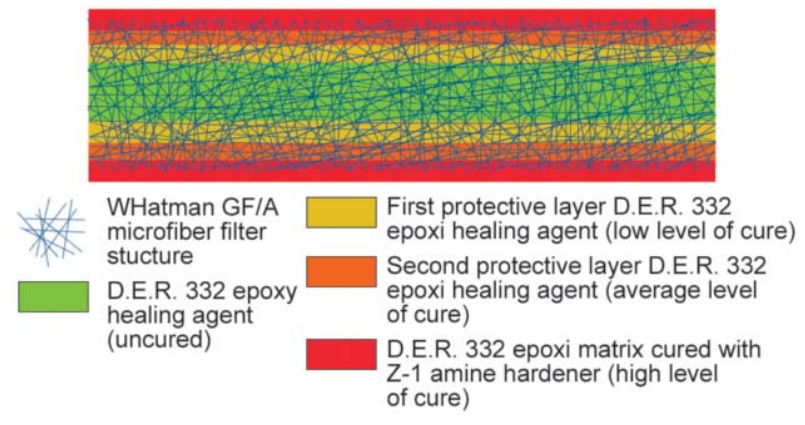

Figure 8. Schematic cross-section of HPGL with protective layers of partially-cured healing agent.

bring the specimen into contact. This is also important for the evaluation of the drops of the healing efficiency, which will be significantly lower for microcracks.

The structure of a glass porous layer provides appropriate isolation of the crystallized healing agent in filter pores. This might result from the synergy of several factors: small diameters of pores; the solid state of healing agent (it hinders the mobility of 
D.E.R. 332 molecules) and thin, protective layers of the partially-cured healing agent on healing porous glass layer surface (they isolate high amount of healing agent embedded deeper in filter pores) (Figure 8).

\section{Conclusions}

The healing potential of three types of healing porous layers was evaluated. Gravimetric analysis with DSC, liquid chromatography and FT-IR spectra clearly showed that the healing porous glass layer based on Whatman GF/A glass microfiber filters could be a useful carrier for thermally induced self-healing epoxy laminates. Microcrack propagation creates empty spaces, which combined with higher temperature of the healing process provide a good healing agent transport. Also glass fibers, being entangled deep inside HPGL are more flexible than common microcontainers - each flow of the healing agent during self-healing might cause them to change position and consequently provide better distribution of the remaining healing agent in HPGL. This property gives HPGL an advantage over other extrinsic self-healing systems and makes it possible to recover the structure few times after serious damage. In conclusion, the obtained thermally induced self-healing laminates with HPGL and woven glass fiber mats reinforcement are characterized with good mechanical properties and competitive self healing ability.

\section{References}

[1] White S. R., Sottos N. R., Geubelle P. H., Moore J. S., Kessler M., Sriram S. R., Brown E. N., Viswanathan S.: Autonomic healing of polymer composites. Nature, 409, 794-797 (2001). https://doi.org/10.1038/35057232

[2] Dry C.: Procedures developed for self-repair of polymer matrix composite materials. Composite Structures, 35, 263-269 (1996). https://doi.org/10.1016/0263-8223(96)00033-5

[3] Toohey K. S., Sottos N. R., Lewis J. A., Moore J. S., White S. R.: Self-healing materials with microvascular networks. Nature Materials, 6, 581-585 (2007). https://doi.org/10.1038/nmat1934

[4] Moll J. L., White S. R., Sottos N. R.: A self-sealing fiberreinforced composite. Journal of Composite Materials, 44, 2573-2585 (2010).

https://doi.org/10.1177/0021998309356605

[5] Pang J. W., Bond I. P.: A hollow fibre reinforced polymer composite encompassing self-healing and enhanced damage visibility. Composites Science and Technology, 65, 1791-1799 (2005).

https://doi.org/10.1016/j.compscitech.2005.03.008
[6] Zako M., Takano N.: Intelligent material systems using epoxy particles to repair microcracks and delamination damage in GFRP. Journal of Intelligent Material Systems and Structures, 10, 836-841 (1999).

https://doi.org/10.1106/YEIH-QUDH-FC7W-4QFM

[7] Yao Y., Wang J., Lu H., Xu B., Fu Y., Liu Y., Leng J.: Thermosetting epoxy resin/thermoplastic system with combined shape memory and self-healing properties. Smart Materials and Structures, 25, 015021/1-015021/9 (2015).

https://doi.org/10.1088/0964-1726/25/1/015021

[8] Karger-Kocsis J.: Self-healing properties of epoxy resins with poly( $\varepsilon$-caprolactone) healing agent. Polymer Bulletin, 73, 3081-3093 (2016). https://doi.org/10.1007/s00289-016-1642-2

[9] Karger-Kocsis J., Kéki S.: Review of progress in shape memory epoxies and their composites. Polymers, 10, $34 / 1-34 / 38$ (2017).

https://doi.org/10.3390/polym10010034

[10] Trask R. S., Bond I. P.: Biomimetic self-healing of advanced composite structures using hollow glass fibres. Smart Materials and Structures, 15, 704-710 (2006). https://doi.org/10.1088/0964-1726/15/3/005

[11] Zhu Y., Ye X. J., Rong M. Z., Zhang M. Q.: Self-healing glass fiber/epoxy composites with polypropylene tubes containing self-pressurized epoxy and mercaptan healing agents. Composites Science and Technology, 135, 146-152 (2016). https://doi.org/10.1016/j.compscitech.2016.09.020

[12] Zanjani J. S. M., Okan B. S., Yilmaz C., Menceloglu Y., Yildiz M.: Monitoring the interface and bulk selfhealing capability of tri-axial electrospun fibers in glass fiber reinforced epoxy composites. Composites Part A: Applied Science and Manufacturing, 99, 221-232 (2017).

https://doi.org/10.1016/j.compositesa.2017.04.017

[13] Bekas D. G., Baltzis D., Paipetis A. S.: Nano-reinforced polymeric healing agents for vascular self-repairing composites. Materials and Design, 116, 538-544 (2017). https://doi.org/10.1016/j.matdes.2016.12.049

[14] Kostopoulos V., Kotrotsos A., Tsantzalis S., Tsokanas P., Loutas T., Bosman A. W.: Toughening and healing of continuous fibre reinforced composites by supramolecular polymers. Composites Science and Technology, 128, 84-93 (2016). https://doi.org/10.1016/j.compscitech.2016.03.021

[15] Lu L., Pan J., Li G.: Recyclable high-performance epoxy based on transesterification reaction. Journal of Materials Chemistry A, 5, 21505-21513 (2017). https://doi.org/10.1039/C7TA06397K

[16] Post W., Cohades A., Michaud V., van der Zwaag S., Garcia S. J.: Healing of a glass fibre reinforced composite with a disulphide containing organic-inorganic epoxy matrix. Composites Science and Technology, 152, 8593 (2017).

https://doi.org/10.1016/j.compscitech.2017.09.017 
[17] Brown E. N.: Use of the tapered double-cantilever beam geometry for fracture toughness measurements and its application to the quantification of self-healing. The Journal of Strain Analysis for Engineering Design, 46, 167-186 (2011)

https://doi.org/10.1177/0309324710396018

[18] Wool R. P., O'Connor K. M.: A theory crack healing in polymers. Journal of Applied Physics, 52, 5953-5963 (1981).

https://doi.org/10.1063/1.328526

[19] Urbaniak M.: A relationship between the glass transition temperature and the conversion degree in the curing reaction of the EPY epoxy system. Polimery, 56, 240-243 (2011).

[20] Schawe J., Riesen R., Widmann J., Schubnell M., Jörimann U.: Interpreting DSC curves. Part 1: Dynamic measurements. Mettler Toledo UserCom, 1-28 (2000).
[21] Sales R. C. M., Diniz M. F., Dutra R. C. L., Thim G. P., Dibbern-Brunelli D.: Study of curing process of glass fiber and epoxy resin composite by FT-NIR, photoacoustic spectroscopy and luminescence spectroscopy. Journal of Materials Science, 46, 1814-1823 (2011). https://doi.org/10.1007/s10853-010-5005-3

[22] Nikolic G., Zlatkovic S., Cakic M., Cakic S., Lacnjevac C., Rajic Z.: Fast fourier transform IR characterization of epoxy GY systems crosslinked with aliphatic and cycloaliphatic EH polyamine adducts. Sensors, 10, 684696 (2010). https://doi.org/10.3390/s100100684

[23] Barbero E. J., Greco F., Lonetti P.: Continuum damagehealing mechanics with application to self-healing composites. International Journal of Damage Mechanics, 14, 51-81 (2005). https://doi.org/10.1177/1056789505045928

[24] Privman V., Dementsov A., Sokolov I.: Modeling of selfhealing polymer composites reinforced with nanoporous glass fibers. Journal of Computational and Theoretical Nanoscience, 4, 190-193 (2007).

https://doi.org/10.1166/jctn.2007.022 